\title{
Myocardial Infarction Does Not Accelerate Atherosclerosis in a Mouse Model of Type 1 Diabetes
}

\author{
Farah Kramer, ${ }^{1}$ Amy M. Martinson, ${ }^{2}$ Thalia Papayannopoulou, ${ }^{3}$ and Jenny E. Kanter ${ }^{1}$
}

Diabetes 2020;69:2133-2143 | https://doi.org/10.2337/db20-0152

In addition to increasing the risk of an initial myocardial infarction (MI), diabetes increases the risk of a recurrent MI. Previous work suggests that an experimental MI can accelerate atherosclerosis via monocytosis. To test whether diabetes and experimental MI synergize to accelerate atherosclerosis, we performed ligation of the left anterior descending coronary artery to induce experimental MI or sham surgery in nondiabetic and diabetic mice with preexisting atherosclerosis. All mice subjected to experimental MI had significantly reduced left ventricular function. In our model, in comparisons with nondiabetic sham mice, neither diabetes nor MI resulted in monocytosis. Neither diabetes nor MI led to increased atherosclerotic lesion size, but diabetes accelerated lesion progression, exemplified by necrotic core expansion. The necrotic core expansion was dependent on monocyte recruitment, as mice with myeloid cells deficient in the adhesion molecule integrin $\alpha 4$ were protected from necrotic core expansion. In summary, diabetes, but not $\mathrm{MI}$, accelerates lesion progression, suggesting that the increased risk of recurrent MI in diabetes is due to a higher lesional burden and/or elevated risk factors rather than the acceleration of the underlying pathology from a previous MI.

Diabetes increases the risk of atherosclerotic cardiovascular disease (1-4). In addition to increasing the risk of an initial myocardial infarction (MI), diabetes also increases the risk of a recurrent MI (5-8). The mechanisms whereby this happens are unknown.

In experimental model systems, an acute experimental MI under nondiabetes conditions can accelerate the underlying atherosclerotic process (9). Accelerated atherosclerosis observed in response to the acute ischemic event was postulated to be driven by enhanced extramedullary hematopoiesis resulting in increased levels of circulating monocytes available for recruitment into the nascent atherosclerotic lesion, thereby accelerating atherosclerosis (9).

Diabetes accelerates atherosclerosis lesion initiation and progression and hinders lesion regression in response to dramatic lipid lowering (10-13). Changes in monocyte and macrophage phenotype are believed to contribute to the acceleration of atherosclerosis in diabetes. In both mouse and human studies, diabetes results in increased macrophage accumulation within the artery wall $(10,14,15)$. For example, autopsy and atherectomy samples from humans have shown that lesions from subjects with diabetes have increased macrophage accumulation compared with specimens from patients without diabetes (16). Studies of mouse models of diabetes-accelerated atherosclerosis have revealed that arterial accumulation of macrophages is accelerated by diabetes $(11,17)$. What is driving the increase in macrophage accumulation is not fully understood. Increased monocyte recruitment appears to be involved, at least in models of lesion regression (18). Consistent with the notion that recruitment is increased, diabetes is often associated with increased endothelial adhesion molecule expression $(19,20)$, which in part could be explained by reduced insulin signaling in endothelial cells (21). In addition to changes in the endothelium, monocytes isolated from humans with diabetes adhere better to aortic endothelial cells (22). Monocyte recruitment involves initial rolling, adhesion, and, finally, transmigration, all of which are highly regulated steps. One of the critical interactions is between endothelial VCAM-1, which is upregulated in diabetes $(19,20)$, and monocyte very-late antigen 4 (VLA4). VLA4 consists of

\footnotetext{
1Division of Metabolism, Endocrinology and Nutrition, Department of Medicine, University of Washington Medicine Diabetes Institute, University of Washington School of Medicine, Seattle, WA

2Department of Laboratory Medicine and Pathology, University of Washington School of Medicine, Seattle, WA

${ }^{3}$ Division of Hematology, Department of Medicine, University of Washington School of Medicine, Seattle, WA

Corresponding author: Jenny E. Kanter, jenka@uw.edu
}

\section{Received 12 February 2020 and accepted 17 July 2020}

This article contains supplementary material online at https://doi.org/10.2337/ figshare.12670325.

(C) 2020 by the American Diabetes Association. Readers may use this article as long as the work is properly cited, the use is educational and not for profit, and the work is not altered. More information is available at https://www.diabetesjournals .org/content/license. 
integrin $\alpha 4$ (also known as CD49D) and integrin $\beta 1$. Blocking VLA4 using monoclonal antibodies and blocking peptides reduces monocyte and macrophage recruitment to the artery wall in atherosclerosis-prone mice $(23,24)$, and deletion of integrin $\alpha 4$ results in a reduced recruitment of monocytes to an inflamed peritoneum $(25,26)$.

The current study was designed to investigate whether diabetes and experimental MI synergize to accelerate atherosclerosis, potentially explaining the increased risk of recurrent $\mathrm{MI}$ in people with diabetes. We show that neither diabetes nor MI results in monocytosis but that diabetes alone and in the presence of MI results in an expansion of the necrotic core, a hallmark of advanced, unstable lesion phenotypes. Furthermore, the development of the necrotic cores is dependent on integrin $\alpha 4$-mediated monocyte recruitment, highlighting the importance of monocytes and macrophage in diabetes-accelerated atherosclerosis.

\section{RESEARCH DESIGN AND METHODS}

\section{Mouse Models}

The T cell-induced LDL receptor-deficient $\left(L d l r^{-1-}\right) G p^{T g}$ mouse model of type 1 diabetes-accelerated atherosclerosis has previously been described (11). These mice express the lymphocytic choriomeningitis virus (LCMV) glycoprotein under control of the insulin promoter, allowing reliable induction of diabetes due to $\mathrm{CD}^{+}{ }^{+} \mathrm{T}$ cell-mediated $\beta$-cell destruction following LCMV injection. In the current study (Fig. 1A), female $L d l r^{-/-} ; G p^{T g}$ mice on the C57BL/6J background, 6-8 weeks of age, mice were fed a high-fat, semipurified diet $(40 \%$ calories from fat, $1.25 \%$ cholesterol) to initiate preexisting lesions for 12 weeks $(11,13)$ and then switched to standard mouse chow to reduce plasma cholesterol to allow for successful bone marrow reconstitution. The mice were lethally irradiated $(10 \mathrm{~Gy})$ and

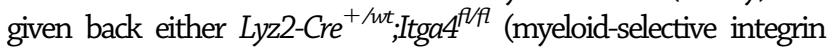
$\alpha$-deficient) (knockout [KO]) or LyzM-Cre ${ }^{+/ w t}$;Itga4 ${ }^{w t / w t}$ (WT) bone marrow and allowed to recover for 5 weeks before one-half of the cohort was rendered diabetic with LCMV. Nondiabetic littermates were injected with saline. At this point, a subset of animals was euthanized, and baseline atherosclerosis was analyzed. At the onset of diabetes (defined as blood glucose levels $>250 \mathrm{mg} / \mathrm{dL}$ ) 10 days after LCMV injection, the nondiabetic and diabetic mice were fed a low-fat semipurified diet without added cholesterol as previously described (11). The low-fat diet was chosen to minimize the plasma lipid differences between diabetic and nondiabetic mice (11). Preliminary data suggest that it results in lesion progression rather than regression (11). The diabetic mice received subcutaneous insulin pellets (LinShin Canada Inc., Toronto, ON, Canada) to provide baseline insulin and were treated with insulin glargine (Lantus; Sanofi, Bridgewater, NJ) to prevent weight loss and ketonuria as needed $(11,15)$. Fourteen days after LCMV injection, the mice were subjected to experimental MI or sham surgery $(27,28)$. Briefly, the mice were orotracheally intubated and mechanically ventilated, a thoracotomy was performed, the pericardium was incised and retracted to expose the heart, and the left coronary artery was permanently ligated with an 8-0 suture. The chest was then closed in layers, and the animal was allowed to recover. The mice in the sham groups were subjected to the same procedure of bar ligation of the artery. The survival rates were $\sim 80 \%$ in both the diabetic and nondiabetic groups, with no difference in survival within the groups subjected to permanent ligation. All mice received buprenorphine analgesia and were monitored regularly until end point analysis. Mice were euthanized 3.5 weeks after the surgery. Four days before euthanasia, the mice were injected retro-orbitally with $250 \mu \mathrm{L}$ yellow-green (YG) latex microparticles (diluted 1:4 in sterile PBS) for monocyte tracing (Fluoresbrite YG Microspheres $0.5 \mu \mathrm{m}$; Polysciences, via Thermo Fisher Scientific) (see Supplementary Table 1). For the analysis of monocyte recruitment, the number of beads per lesion was normalized to the number of labeled monocytes in circulation. At the end of the experiments, macrophages were isolated from the peritoneal cavity, using ice-cold PBS + $5 \mathrm{mmol} / \mathrm{L}$ EDTA. The macrophages were allowed to adhere for $1 \mathrm{~h}$ before they were harvested for either RNA isolation (NucleoSpin RNA Plus; MACHEREY-NAGEL, Bethlehem, PA) or measurements of total cholesterol, free cholesterol, and cholesteryl esters (Amplex Red Cholesterol Assay Kit; Thermo Fisher Scientific, Grand Island, NY). Additional control groups included mice that were not bone marrow transplanted and had not had prior fat feeding, mice that did not express the viral glycoprotein but were injected with LCMV, and a comparison cohort of male and female nondiabetic and diabetic mice that had been fat fed but not bone marrow transplanted.

\section{Echocardiogram}

One day prior to euthanasia, mice were subjected to echocardiography using a VisualSonics Vevo 2100. The left ventricular function was assessed in M-mode at the level where the papillary muscle could be observed during isoflurane sedation (mean heart rate $430 \mathrm{bpm}$ ). Data were analyzed by an investigator blinded to the study groups.

\section{Analysis of Glucose and Lipid Metabolism}

Blood glucose was measured in the saphenous vein blood by stick tests (OneTouch Ultra). As the glucometer does not go beyond $600 \mathrm{mg} / \mathrm{dL}$, values above that are set to $600 \mathrm{mg} / \mathrm{dL}$. Plasma cholesterol levels were determined by the Cholesterol E kit (Wako Diagnostics, Wako, TX), and plasma triglycerides were determined by a colorimetric kit from Sigma-Aldrich.

\section{Quantification of Atherosclerosis and Infarction Area}

The aorta was dissected after in situ perfusion with PBS and was fixed in $10 \%$ phosphate-buffered formalin (SigmaAldrich). Aortas were opened longitudinally (from the heart to the iliac bifurcation) and en face after Sudan IV staining was quantified, as previously described $(11,15)$. Brachiocephalic arteries (BCAs) and aortic sinuses were cross-sectioned and stained with the Movat pentachrome 

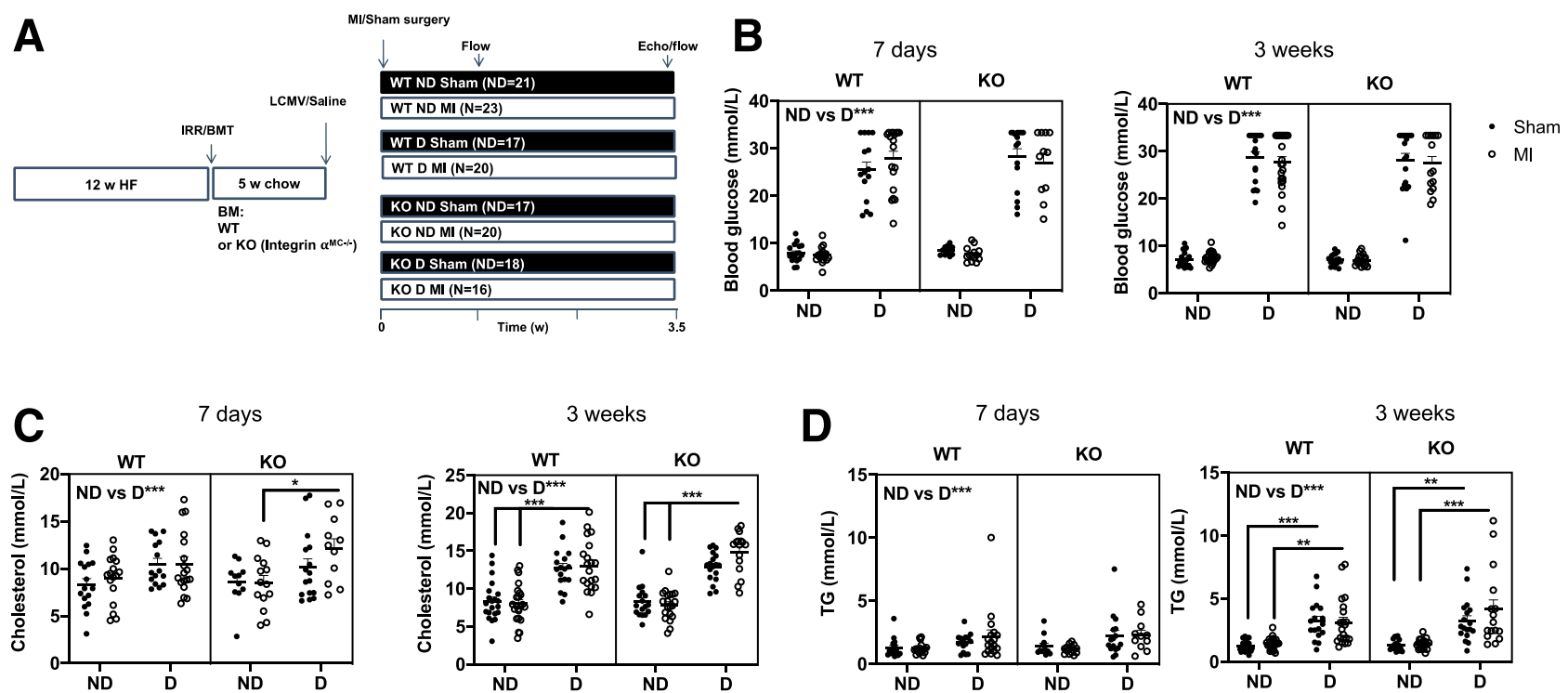

Figure 1-Experimental MI does not affect diabetic hyperglycemia or dyslipidemia. $A$ : Schematic of the study plan. Female $L d l r^{-/-} ; G p^{T g}$ mice were fed a high-fat (HF) diet with $1.25 \%$ cholesterol for 12 weeks (w) to initiate lesion formation, switched to chow (to allow for successful bone marrow $[\mathrm{BM}]$ reconstitution), and lethally irradiated and transplanted with either WT or LysM-mediated KO bone marrow and allowed to recover for 5 weeks before injection with LCMV to induce diabetes (D) or saline (nondiabetic [ND]). Two weeks post-LCMV injection, mice were subjected to permanent ligation of the left anterior descending coronary artery (experimental $\mathrm{MI}$ ) or a sham surgery and maintained for an additional 3.5 weeks. Mice were maintained on a low-fat semipurified diet from the onset of diabetes. $B$ : Blood glucose at day 7 and at 3 weeks. C: Plasma cholesterol on day 7 and at 3 weeks. $D$ : Plasma triglycerides (TG) at day 7 and at 3 weeks. $N=15-23$. ${ }^{*} P<0.05$, ${ }^{\star *} P<$ $0.01,{ }^{* \star \star} P<0.001$, three-way ANOVA (WT vs. KO, nondiabetic vs. diabetic, sham vs. Ml as the parameters, or the interaction between these) followed by Sidak multiple comparisons tests comparing groups that differ by one parameter (as indicated by lines with asterisks). The differences between nondiabetic and diabetic mice are not shown with lines in $B$ (all $\left.{ }^{\star \star \star}\right)$. BMT, bone marrow transplant; IRR, irradiation.

stain to visualize lesion morphology. The aortic sinus was analyzed at the site of all three leaflets. The heart was serially sectioned, and every $30 \mu \mathrm{m}$ was stained with Movat pentachrome stain. The size of the infarction was measured as the largest infarction in the left ventricle. Lesional macrophages and smooth muscle cells were visualized by Mac-2 and $\alpha$-smooth muscle actin immunohistochemistry, respectively (13) (Supplementary Table 1). Lesions were also stained for TREM2, IL-1 $\beta$, and Picrosirius red (to identify collagen) (Polysciences). All histological analyses were performed by an investigator blinded to the treatment groups.

\section{Flow Cytometry of Blood Leukocytes and Peritoneal Macrophages}

Mice were bled from the retro-orbital plexus under isoflurane sedation 1 week after surgery and 1 day prior to euthanasia. EDTA was used as anticoagulant. Total leukocytes were measured using an automated cell counter for mouse blood samples (HEMAVET; Drew Scientific, Oxford, CT). For flow cytometric analysis, erythrocytes were lysed with an ammonium-chloride-potassium buffer and discarded, and leukocytes were stained using a fixable viability dye, CD45, CD115, and GR1 (see Supplementary Table 1). Monocytes were identified as $\mathrm{CD} 115^{+}$cells, and neutrophils were identified as $\mathrm{CD} 115^{-} \mathrm{GR} 1^{\text {hi }}$ cells and gated as in Supplementary Fig. 2. All analyses were performed with live, single, $\mathrm{CD} 45^{+}$cells. The monocytes were further divided into $\mathrm{GR}^{\text {hi }}$ (Ly6C $\mathrm{Ch}^{\text {hi }}$ ) and $\mathrm{GR} 1^{\text {lo }}\left(\mathrm{Ly}_{6 \mathrm{C}} \mathrm{C}^{\mathrm{lo}}\right.$ ) subpopulations. Flow cytometric analysis was normalized to total white blood cell (WBC) counts and expressed as cells per milliliter of blood, using the assumption that all $\mathrm{WBC}$ are $\mathrm{CD} 45^{+}$. A portion of the peritoneal exudate was stained with fixable viability dye and CD11B and F480 (Supplementary Table 1). The samples were gated as described in Supplementary Fig. 8. The cells were analyzed on a BD FACS RUO flow cytometer (BD Biosciences, Franklin Lakes, NJ).

\section{Ex Vivo Monocyte Adhesion}

Monocytes were isolated from bone marrow from a subset of animals from the main study using the EasySep Mouse Monocyte Isolation Kit (STEMCELL Technologies, Seattle, WA). Each mouse $=1 \mathrm{n}$. Monocytes were stained with Calcein AM (1 $\mu \mathrm{g} / \mathrm{mL}$ ) (Invitrogen, Carlsbad, CA) for 30 min, washed, and then added to primary mouse microvascular endothelial cells (29) grown in a 96-well plate at 150,000 monocytes/well and allowed to adhere for $30 \mathrm{~min}$. The nonadherent monocytes were then washed off with PBS and the remaining cells were lysed, and the lysate was then transferred to a black plate and read using a plate reader capable of reading 485/528 nm.

\section{Real-time PCR}

Gene expression in resident peritoneal macrophages was quantified by real-time PCR. RNA isolation and the real-time 
PCR protocol were performed as previously described (15). Briefly, RNA was isolated using MACHEREY-NAGEL NucleoSpin RNA kits with on-column DNase treatment according to the manufacturers' protocols. Real-time PCR was performed using the SYBR Green 1 detection method (Thermo Fisher Scientific). Cycle threshold values were normalized to $\mathrm{Rn} 18 \mathrm{~s}$ and presented as fold over control. Primer sequences are listed in Supplementary Table 2.

\section{Statistical Analyses}

Statistical analyses of the mouse data were performed using GraphPad Prism 8.0 (GraphPad Software, La Jolla, CA). To compare four groups, we used a two-way ANOVA followed by Tukey multiple comparisons test. For comparisons between all eight experimental groups, a three-way ANOVA was used, comparing nondiabetic with diabetic, WT with KO, and sham with MI. Significant differences are indicated for nondiabetic vs. diabetic, WT vs. KO, and/or sham vs. MI significance or as an interaction between two conditions (e.g., DxKO indicates that under diabetic conditions there is an effect of the KO). This allows us to assess the overall effect of diabetes, integrin $\alpha$ deficiency (KO), and MI. The three-way ANOVA was followed by the Sidak multiple comparisons test comparing all groups but only those that differed by one factor (e.g., in WT mice under sham conditions, nondiabetic versus diabetic). These statistical differences are indicated with lines between the different groups. A $P$ value of $<0.05$ was considered statistically significant.

\section{Data and Resource Availability}

The majority of the data generated or analyzed during this study are included here and in the supplementary material, but additional data sets generated during and/or analyzed during the current study are available from the corresponding author upon reasonable request. The resources generated during and/or analyzed during the current study are available from the corresponding author upon reasonable request.

\section{RESULTS}

\section{Experimental MI in Diabetic Mice Does Not Worsen Diabetes}

To test whether experimental MI and diabetes synergize to accelerate atherosclerosis, we took advantage of our inducible type 1 diabetes model $(11-13,15)$. To this end, we initiated lesion formation by fat feeding the mice for 12 weeks (Fig. 1A). At this point, the mice were bone marrow transplanted with either WT ( LyzM-Cre ${ }^{+/ w t}$;Itga $\left.4^{w t / w t}\right)$ or KO (myeloid-selective integrin $\alpha 4$-deficient $\left(\right.$ Lyz2-Cre $^{+/ w t} ;$ Itga $4^{f / / f}$ ) bone marrow. Integrin $\alpha 4$ plays a crucial role in regulating leukocyte entry into tissues (30), and together with integrin $\beta 1$, integrin $\alpha 4$ makes up VLA4, which binds VCAM-1 on activated endothelial cells (30). Following the bone marrow transplant, the mice were allowed to recover for 5 weeks before diabetes was induced in one-half of the cohort. Following the onset of diabetes, one-half of each cohort was then subjected to an experimental MI, which involved permanent ligation of the left descending coronary artery. The other one-half was subjected to sham surgery. Importantly, neither induction of MI nor bone marrow genotype affected the onset or severity of diabetes, as blood glucose levels were similar between the diabetic groups both 7 days postsurgery and 3 weeks after (Fig. 1B). Similar to what we have reported previously (12), diabetes results in a modest increase in total cholesterol (Fig. 1C) and elevated plasma triglyceride levels (Fig. 1D), most likely reflecting an increase in triglyceride-rich lipoproteins (12). The elevated lipid levels were evident at both 7 days and 3 weeks post-MI, but neither presence of an ischemic event nor bone marrow genotype affected the level of dyslipidemia.

Importantly, all mice subjected to experimental MI had a similar extent of left ventricular infarcted area (Fig. $2 \mathrm{~A}$ and $B$ ). This translated into a significant reduction in both ejection fraction and fractional shortening 3 weeks after infarction, with no significant differences in impairment between the different groups (Fig. $2 C$ and $D$ ). Consistent with diabetes resulting in cardiomyopathy (31), mild impairment in the left ventricular function was observed in mice with diabetes even in the absence of experimental MI (Fig. $2 C$ and $D$ ).

\section{Diabetes and Diabetes Combined With an Experimental MI Result in Neutrophilia but Not Monocytosis}

A previous MI increases the risk of a recurrent event (6). Dutta et al. (9) proposed a compelling model whereby the initial MI accelerates the atherosclerotic process, thereby increasing the risk of another MI. They demonstrated that experimental MI in a nondiabetic APOE-deficient mice accelerated atherosclerosis via enhanced monocytosis, which in turn resulted in increased macrophage accumulation within the atherosclerotic lesion (9). To investigate whether differences in circulating leukocyte populations might contribute to the acceleration of atherosclerosis after an experimental MI in our experimental cohorts, we evaluated leukocyte populations in the experimental mice at two separate time points. Using an automated WBC counter, we observed no leukocytosis in response to experimental MI either 7 days following infarction or 3 weeks post-MI (Fig. 3A). Diabetes resulted in significant suppression of total WBCs, irrespective of myeloid cell genotype (Fig. $3 A$ ). This reduction in $\mathrm{WBC}$ s was primarily driven by a marked decrease in lymphocytes, which were reduced by $\sim 55 \%$ in diabetes (Supplementary Fig. $2 A$ ). Diabetes resulted in a smaller absolute reduction in neutrophils and monocytes in mice with WT bone marrow, which was reversed in mice with $L y z 2-C r e^{+/ w t} ; \operatorname{Itga} 4^{f / A l}$ bone marrow (Supplementary Fig. $2 B$ and $C$ ). Consistent with the reduction in total WBCs, the spleens were also significantly smaller under diabetes conditions (Supplementary Fig. 2D).

For further evaluation of the levels of circulating cells, mice from all experimental groups were subjected to 
A
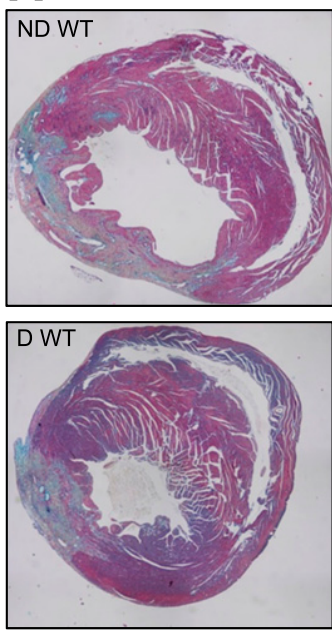

C

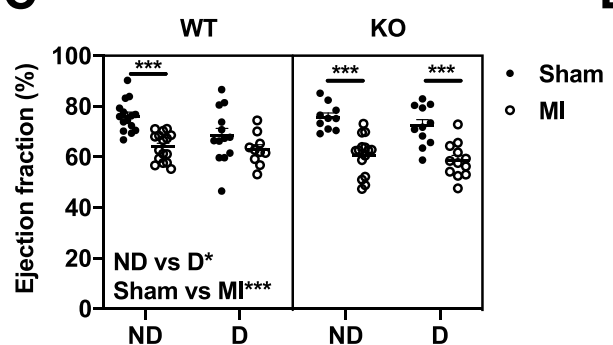

B
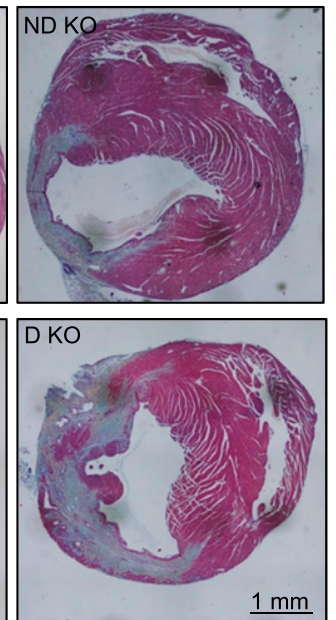
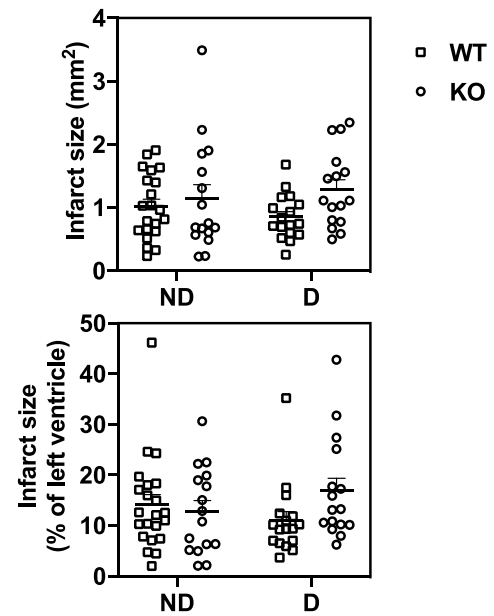

KO

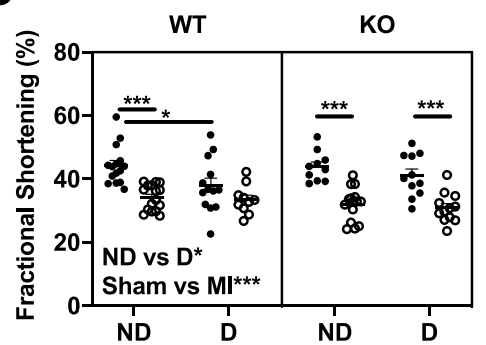

Figure 2-Experimental MI results in reduced cardiac function. Female $L d l r^{-1-} ; G p^{T g}$ mice were fed a high-fat diet with $1.25 \%$ cholesterol for 12 weeks to initiate lesion formation, switched to chow, and lethally irradiated and transplanted with either WT or LysM-mediated KO bone marrow and allowed to recover for 5 weeks before injection with LCMV to induce diabetes (D) or saline (nondiabetic [ND]). Two weeks postLCMV injection, mice were subjected to permanent ligation of the left anterior descending coronary artery (experimental MI) or a sham surgery and maintained for an additional 3.5 weeks. Mice were maintained on a low-fat semipurified diet from the onset of diabetes. One day prior to euthanasia, mice were subjected to echocardiography using a VisualSonic Vevo 2100. Left ventricular function was assessed in M-mode at the level where the papillary muscle could be observed during isoflurane sedation (mean heart rate $430 \mathrm{bpm}$ ). $A$ : Example of cross-sections of the left ventricle stained with Movat pentachrome stain. $B$ : The infarction area expressed as total $\mathrm{mm}^{2}$ or $\%$ of the left ventricle. $C$ : Ejection fraction. $D$ : Fractional shortening. $N=10-16 .{ }^{*} P<0.05,{ }^{\star *} P<0.01,{ }^{* \star *} P<0.001$, two-way ANOVA $(B)$ and three-way ANOVA (C and $D$ [WT vs. $\mathrm{KO}$, nondiabetic vs. diabetic, sham vs. $\mathrm{Ml}$ as the parameters, or the interaction between these]) followed by Sidak multiple comparisons tests comparing groups that differ by one parameter (as indicated by lines with asterisks).

flow cytometric analysis (Supplementary Fig. $1 A$ and $B$ ). Consistent with adequate bone marrow reconstitution after the bone marrow transplantation, monocytes from mice

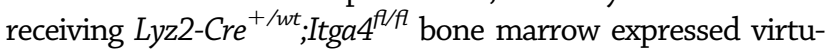
ally no integrin $\alpha 4$ on their cell surface (Fig. $3 B$ and Supplementary Fig. 1A). There was a $92 \%$ reduction in cell surface integrin $\alpha 4$ expression in monocytes reconstituted with Lyz2-Cre ${ }^{+/ w t}$;Itga4 $4^{f / A}$ bone marrow compared with mice reconstituted with WT bone marrow (Lyz2-Cre $\left.{ }^{+/ w t} ; \operatorname{Itga} 4^{w t / w t}\right)$. Interestingly, diabetes resulted in an upregulation of monocyte integrin $\alpha 4$ (Fig. 3B). In mice, there are two major populations of monocytes: Ly6 $\mathrm{C}^{\mathrm{hi}}$ and Ly6 $\mathrm{C}^{\mathrm{lo}}$ monocytes. There was a similar reduction in cell surface integrin $\alpha 4$ expression in both monocyte populations (90\% and $96 \%$ in the $\mathrm{Ly} 6 \mathrm{C}^{\mathrm{hi}}$ and the $\mathrm{Ly} 6 \mathrm{C}^{\mathrm{lo}}$ population, respectively). Lysosome M (LysM)-mediated deletion of integrin $\alpha 4$ could also be detected in neutrophils ( $\sim 55 \%$ reduction), albeit less of a reduction compared with that in monocytes (Supplementary Fig. 3A). The reduced integrin $\alpha 4$ expression on both monocytes and neutrophils is consistent with the known activation of the LysM promoter in both monocytes/macrophages and neutrophils (32).

Elevated circulating levels of monocytes can be a potent driver of atherosclerosis $(9,18)$. Diabetes has been reported to result in monocytosis and thereby contribute to diabetesassociated atherogenesis in mouse models (18); however, we typically do not observe increased circulating monocyte levels in our type 1 diabetes mouse model even when atherosclerosis is accelerated by diabetes $(12,33)$. Consistent with our previous findings, diabetes did not result in monocytosis (Fig. 3C and Supplementary Fig. 2C). Instead, at least at the earlier time point, diabetes significantly suppressed circulating monocyte levels. This suppression was evident in both Ly6 $C^{\text {hi }}$ and Ly6 $6 C^{\text {low }}$ monocyte populations (Supplementary Fig. $3 B$ and $C$ ) but absent in mice with monocyte deficiency in integrin $\alpha 4$ (Fig. $3 C$ and Supplementary Fig. $3 B$ and $C$ ), consistent with the notions of integrin $\alpha 4$ being critical for recruitment of monocytes into 

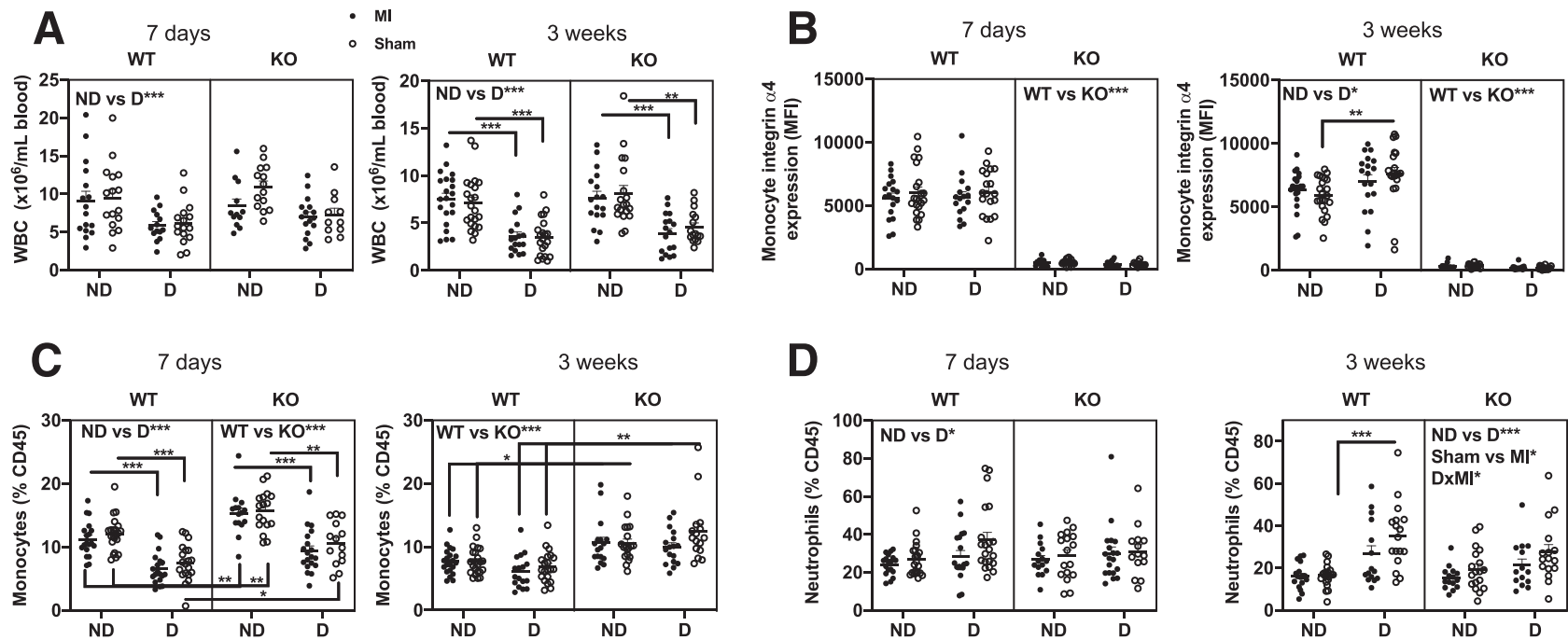

Figure 3-Diabetes results in neutrophilia but not monocytosis. Female $\mathrm{Ldll}^{-1-} ; G \mathrm{p}^{T g}$ mice were fed a high-fat diet with $1.25 \%$ cholesterol for 12 weeks to initiate lesion formation, switched to chow, and lethally irradiated and transplanted with either WT or LysM-mediated KO bone marrow and allowed to recover for 5 weeks before injection with LCMV to induce diabetes (D) or saline (nondiabetic [ND]). Two weeks postLCMV injection, mice were subjected to permanent ligation of the left anterior descending coronary artery (experimental MI) or a sham surgery and maintained for an additional 3.5 weeks. Mice were maintained on a low-fat semipurified diet from the onset of diabetes. $A$ : WBC count at 7 days and the end of the study using a HEMAVET veterinary hematology system. $B$ : Flow cytometric analysis of blood monocytes integrin $\alpha 4$ expression at 7 days and at 3 weeks. C: Monocytes, defined as single, live, CD45 ${ }^{+}$, and CD115 cells at 7 days and at 3 weeks. D: Neutrophils, defined as single, live, $\mathrm{CD} 45^{+}, \mathrm{CD} 115^{-}$, and GR1 ${ }^{+}$cells at 7 days and at 3 weeks. $N=14-22 .{ }^{*} P<0.05,{ }^{\star \star} P<0.01$, ${ }^{\star \star \star} P<$ 0.001 , three-way ANOVA (WT vs. KO, nondiabetic vs. diabetic, sham vs. MI as the parameters, or the interaction between these) followed by Sidak multiple comparisons tests comparing groups that differ by one parameter (as indicated by lines with asterisks). The differences between each WT and KO group are not shown with lines in $B$ (all $\left.{ }^{* \star *}\right)$.

tissues and that diabetes results in increased monocyte recruitment into tissues. If cells fail to enter tissues, they may accumulate in the circulation $(25,26)$. The suppression of monocyte levels could not be attributed to either bone marrow transplantation or effects of the LCMV (Supplementary Table 3).

In contrast to previously published results (9), experimental MI itself did not induce monocytosis either in nondiabetic or diabetic mice compared with mice subjected to sham surgery (Fig. $3 C$ and Supplementary Fig. $2 C$ ). The lack of monocytosis with MI might be explained by differences in models used or experimental setup, as the other study was carried out in APOE-deficient mice and did not include sham controls (34). However, consistent with the idea that diabetes and experimental MI are indeed associated with a state of heightened inflammation, relative levels of circulating neutrophils were elevated in mice with diabetes, with a significant interaction between diabetes and experimental MI in elevating neutrophil levels (Fig. $3 D$ ). Collectively, we observed no evidence of either MI or diabetes resulting in elevated monocyte levels.

\section{Neither Diabetes nor Experimental MI Increases Atherosclerotic Lesion Size, but Diabetes Results in a More Advanced Lesion}

To begin to understand whether an experimental MI and diabetes might act synergistically to accelerate atherosclerosis, we evaluated atherosclerosis at the end of the study.
Atherosclerosis was analyzed at three separate sites: the aorta, the aortic sinus, and the BCA. The total area occupied by Sudan IV-positive lesions was $5.99 \pm 0.57 \%$ (\% Sudan IV-positive area in the aorta) at baseline and $7.61 \pm 1.32 \%$ in the WT nondiabetic sham group. The total lesion area in the aortic sinus was $344,003 \pm 63,60 \mu \mathrm{m}^{2}$ at baseline vs. $396,659 \pm 31,902 \mu \mathrm{m}^{2}$ in the WT nondiabetic sham group, suggesting that the lesions progress rather than regress during the study. At the end of the study, neither diabetes nor MI affected en face aortic lesion size, sinus lesion size, or BCA lesion size (Fig. $4 A-C$ and Supplementary Fig. $4 A-$ $C)$, as expected, as these were short-term studies on preexisting atherosclerotic lesions. The lack of change in lesion size is also in line with our previous work (12). In both the aorta and in the aortic sinus, the lesion size was greater at the end of the study than lesions analyzed from mice at baseline (right before induction of diabetes).

Both mouse and human studies implicate monocytes and macrophages as critical cell types in atherosclerosis associated with diabetes $(10,14,15)$, and increased monocyte recruitment has been implicated in diabetes-accelerated atherosclerosis (18). To test the role of monocyte recruitment, we chose to target integrin $\alpha 4$, which is integral for monocyte binding to VCAM-1 on endothelial cells. As expected, the deletion of integrin $\alpha 4$ resulted in reduced ex vivo adhesion of isolated monocytes to tumor necrosis factor- $\alpha$-activated endothelium (Supplementary Fig. 5A). Deletion of myeloid cell integrin $\alpha 4$ deficiency 

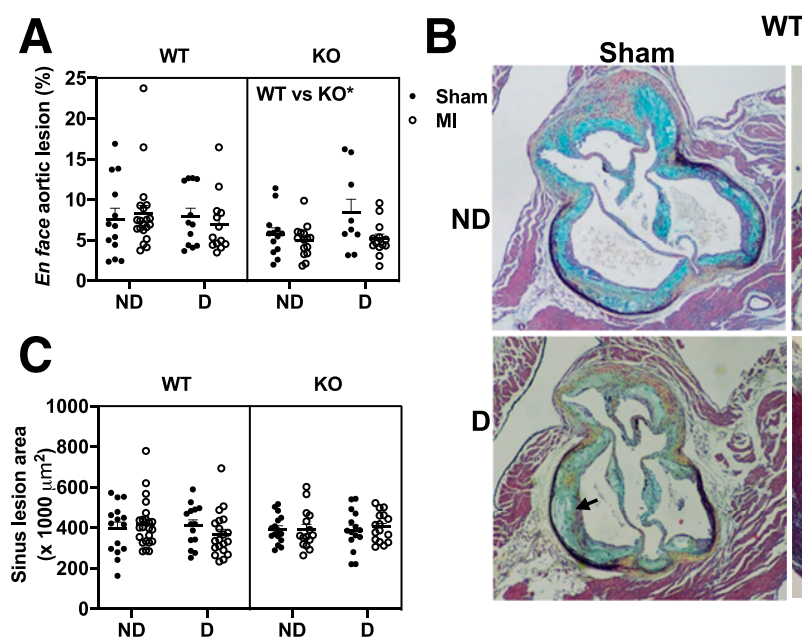

WT
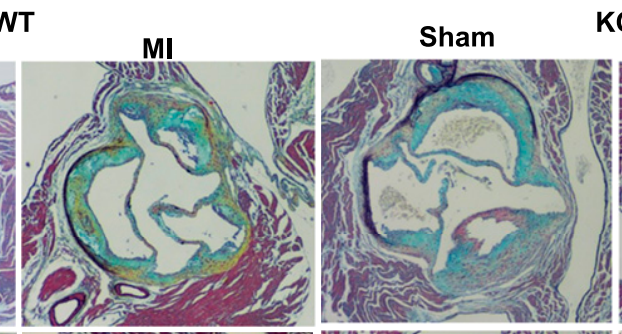

KO
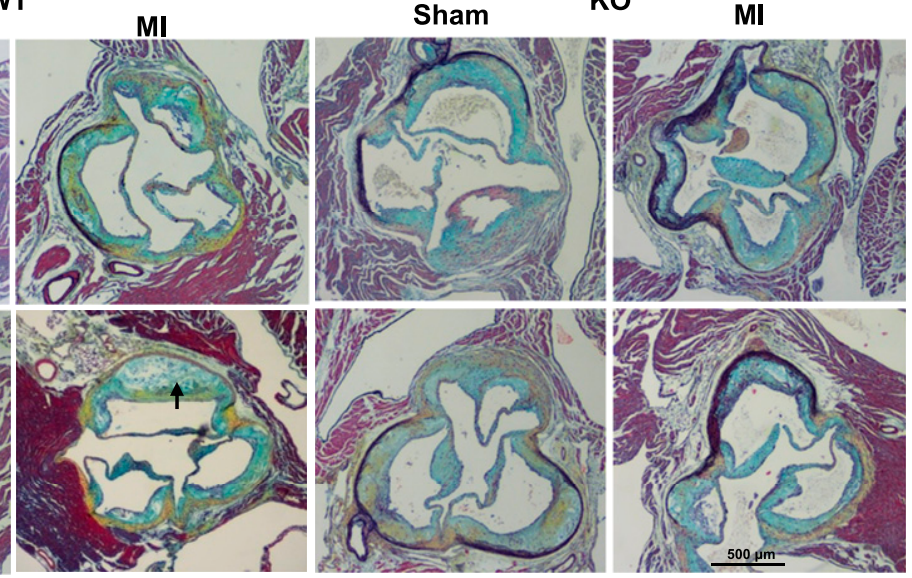

Figure 4-Neither diabetes nor Ml alters lesion size. Female $\mathrm{Ldll}{ }^{-1-} ; G p^{T g}$ mice were fed a high-fat diet with $1.25 \%$ cholesterol for 12 weeks to initiate lesion formation, switched to chow, and lethally irradiated and transplanted with either WT or LysM-mediated KO bone marrow and allowed to recover for 5 weeks before injection with LCMV to induce diabetes (D) or saline (nondiabetic [ND]). Two weeks post-LCMV injection, mice were subjected to permanent ligation of the left anterior descending coronary artery (experimental MI) or a sham surgery and maintained for an additional 3.5 weeks. Mice were maintained on a low-fat semipurified diet from the onset of diabetes. A: Aortic atherosclerosis en face visualized with the lipophilic stain Sudan IV. Quantification of aortic atherosclerosis $(n=9-13)$. B: Example of cross sections from the aortic sinus stained with Movat pentachrome. C: Quantification of the total lesion size in the aortic sinus. Arrows indicate necrotic cores. $N=14-24$. No statistical differences observed, three-way ANOVA (WT vs. KO, nondiabetic vs. diabetic, sham vs. MI as the parameters, or the interaction between these) followed by Sidak multiple comparisons tests comparing groups that differ by one parameter.

resulted in a small protective effect for atherosclerotic lesion size in the aorta (Fig. $4 \mathrm{~A}$ ) but not at either of the other sites. For examination of whether monocyte recruitment was altered under diabetes conditions and whether monocyte integrin $\alpha 4$ deficiency impaired recruitment in vivo, monocytes were labeled with YG latex beads (35) 4 days before termination of the experiment. Bead injections resulted in $\sim 16 \%$ of total monocytes being labeled in nondiabetic WT mice, and the proportion of labeled monocytes, but not the total number of labeled of monocytes, were increased with diabetes (Supplementary Fig. 5B). Monocyte integrin $\alpha 4$ deficiency increased circulating monocyte-labeling efficiency. Furthermore, Ly6 $\mathrm{C}^{\mathrm{lo}}$ monocytes were more efficiently labeled compared with Ly6C ${ }^{\text {hi }}$ monocytes using this approach (Supplementary Fig. 5C), consistent with previously published reports (35). Consistent with the idea that integrin $\alpha 4$ is essential for monocyte adhesion, which in turn is critical for monocyte recruitment, the deletion of monocyte integrin $\alpha 4$ resulted in a reduced recruitment of YG-labeled monocytes into aortic sinus lesions and BCA lesions in vivo (Fig. $5 A$ and $B$ and Supplementary Fig. 5D). Consistent with the increased monocyte integrin $\alpha 4$ expression observed under diabetes conditions (Fig. 3B), diabetes resulted in increased monocyte recruitment to both the aortic sinus and the $\mathrm{BCA}$ (Fig. 5B and Supplementary Fig. 5D).

Despite having significantly more circulating monocytes (Fig. $3 C$ and Supplementary Fig. 2C), mice with monocytes lacking integrin $\alpha 4$ did not have increased lesional Mac-2 staining (Fig. 5C and Supplementary Fig. $5 E$ ), again suggesting that monocytes are not entering the tissues to the same extent in the absence of integrin $\alpha 4$. Furthermore, analysis of the expression of the proatherogenic cytokine IL- $1 \beta$ suggested that under diabetes conditions, the deletion of integrin $\alpha 4$ reduces the accumulation of IL-1 $\beta$ (Fig. $5 D$ ), indicating that integrin $\alpha 4$ deficiency alters the inflammatory status within the lesion due to reduced recruitment of monocytes. In contrast, integrin $\alpha 4$ deletion did not alter the diabetes-induced expression of the marker for lipid loading, TREM2. There was no difference in $\alpha$-smooth muscle actin staining or Picrosirius red staining with MI, diabetes, or integrin $\alpha 4$ deficiency (Supplementary Fig. 5E-G).

To get a fuller picture, we isolated both resident and thioglycolate-elicited peritoneal macrophages from mice receiving WT bone marrow. Diabetes resulted in an increase in total cholesterol and cholesteryl ester accumulation in both resident and thioglycolate-elicited cells, consistent with the elevated TREM2 staining observed in lesions from diabetic mice (Fig. $6 A$ and Supplementary Fig. $6 A$ and $B$ ). Diabetes also increased the expression of proinflammatory cytokines (Fig. 6B), suggesting that macrophages are lipid loaded and more inflammatory under diabetes conditions. Consistent with the notion that diabetes results in augmented inflammatory status, plasma IL18 levels were elevated in diabetes. This increase in IL18 was independent of myeloid cell expression of integrin $\alpha 4$ and whether the mice had been subjected to an MI (Fig. 6C). 


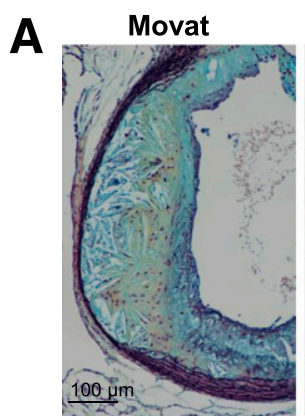

Mac-2

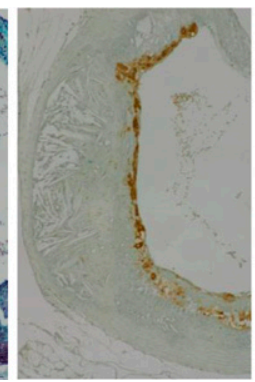

IL-1及

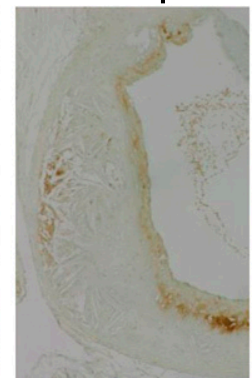

TREM2

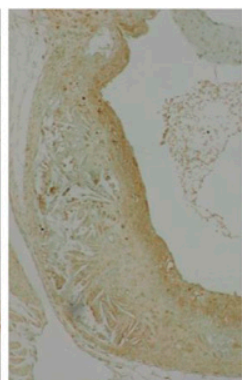

D

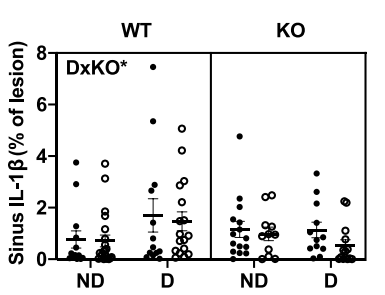

YG Beads

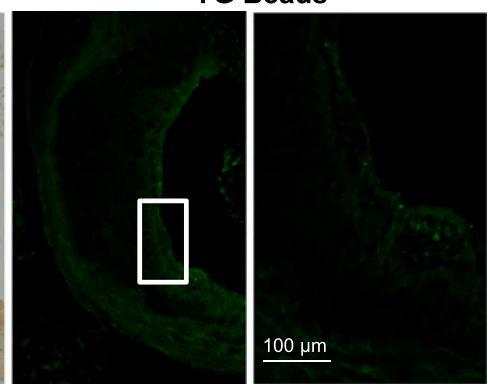

E

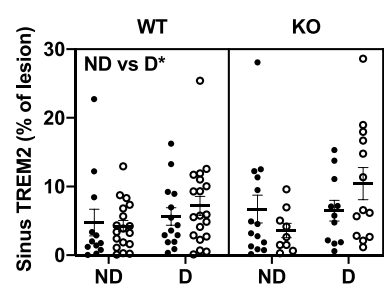

Figure 5-Integrin $\alpha 4$ regulates monocyte recruitment. Female $L d r^{-1-} ; G p^{T g}$ mice were fed a high-fat diet with $1.25 \%$ cholesterol for 12 weeks to initiate lesion formation, switched to chow, and lethally irradiated and transplanted with either WT or LysM-mediated KO bone marrow and allowed to recover for 5 weeks before injection with LCMV to induce diabetes (D) or saline (nondiabetic [ND]). Two weeks post-LCMV injection, mice were subjected to permanent ligation of the left anterior descending coronary artery (experimental MI) or a sham surgery and maintained for an additional 3.5 weeks. Mice were maintained on a low-fat semipurified diet from the onset of diabetes. $A$ : Example of monocyte recruitment to the aortic sinus. From left to right: adjacent sections stained with Movat pentachrome, Mac-2, IL-1 $\beta$, and TREM2 and YG latex beads (indicative of monocytes as described in RESEARCH DESIGN AND METHODS) in the aortic sinus, with the boxed area in higher magnification. $B$ : Monocyte recruitment into the aortic sinus normalized to labeling efficiency. $C$ : Mac-2 area in the aortic sinus expressed as \% lesion. $D: I L-1 \beta$ area in the aortic sinus expressed as \% lesion. $E$ : TREM2 area in the aortic sinus expressed as \% lesion. $N=11-18,{ }^{*} P<0.05$, ${ }^{\star \star \star} P<0.001$ three-way ANOVA (WT vs. KO, nondiabetic vs. diabetic, sham vs. Ml significance or as a significant interaction between two conditions [e.g., DxKO indicates that under diabetic conditions there is an effect of the KO]) and followed by Sidak multiple comparisons tests comparing groups that differ by one parameter (as indicated by lines with asterisks).

Human imaging studies suggest that diabetes accelerates lesion progression, especially the expansion of necrotic cores $(36,37)$. In line with the data on human lesions, we have previously demonstrated that diabetes results in increased necrotic cores in a mouse model of diabetes (12). Dutta et al. (9) reported that experimental MI led to larger necrotic cores. To this end, we investigated whether diabetes and MI might alter necrotic core size. In our hands, experimental MI did not result in a statistically significant expansion of the necrotic cores. However, similar to our previous results, diabetes resulted in an expansion of the necrotic core in the aortic sinus and the BCA (Fig. $6 D$ and $E$ and Supplementary Fig. 7A-C). Although this feature appeared exaggerated with the combination of diabetes and experimental MI, it did not reach statistical significance. Also, flow cytometric analysis of resident peritoneal, thioglycolate-elicited, and spleen F4/ 80 and $\mathrm{CD}_{11 \mathrm{~B}^{+}}$macrophages revealed that diabetes increases cell death in this population (Fig. $6 F$ and Supplementary Figs. $6 C$ and $D$ and 8), potentially contributing to the increased necrotic core size observed in diabetes. Interestingly, the expansion of the necrotic core found under diabetes conditions depended on myeloid cell integrin $\alpha 4$ expression, clearly suggesting that necrotic core expansion under diabetes condition depends on monocyte recruitment.

\section{DISCUSSION}

Here we demonstrate that diabetes accelerates the progression of more advanced lesions, highlighted by an expansion of the necrotic core. Contrary to a previous publication (9), experimental MI in our model did not accelerate atherosclerosis or the expansion of the necrotic core compared with sham surgery. Furthermore, experimental MI also did not further accelerate atherosclerosis under diabetes conditions. The effect of diabetes on necrotic core expansion was dependent on integrin $\alpha 4$ on monocytes, suggesting that monocyte recruitment is critical for the necrotic core to expand. Still, it was not dependent on elevated levels of monocytes in circulation.

Acute events such as an experimental MI have been proposed to accelerate atherosclerosis in APOE-deficient mice, in part explaining the increased risk of subsequent MIs after the initial event (9). The proposed model included activation of the sympathetic nervous system resulting in extramedullary hematopoiesis and elevated monocytes in circulation (9). However, Hoffman et al. (34) demonstrated that a sham surgery induces a level of monocytosis similar 

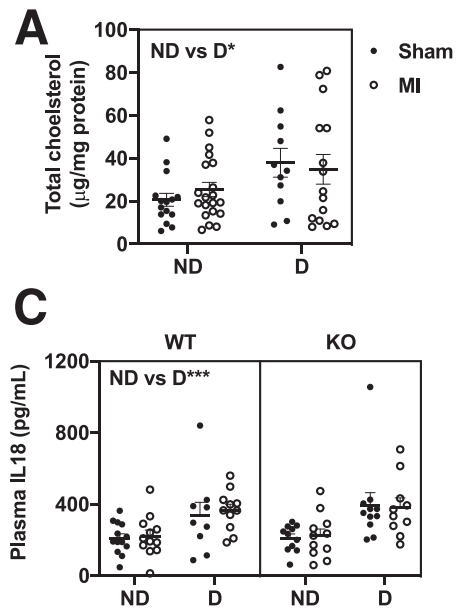
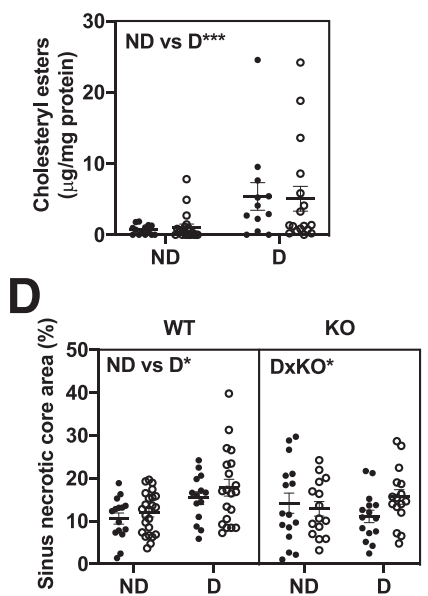

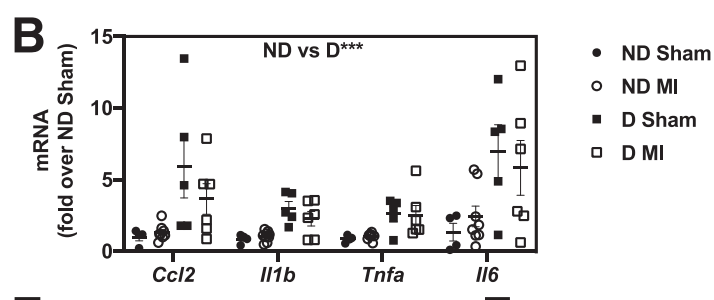

E

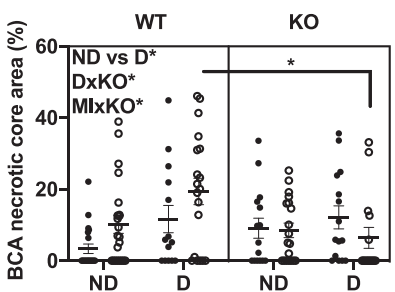

Figure 6-Diabetes increased macrophage cell death and necrotic core size. Female $L d l r^{-1-} ; G p^{T g}$ mice were fed a high-fat diet with $1.25 \%$ cholesterol for 12 weeks to initiate lesion formation, switched to chow, and lethally irradiated and transplanted with either WT or LysMmediated KO (bone marrow and allowed to recover for 5 weeks before injection with LCMV to induce diabetes (D) or saline (nondiabetic [ND]). Two weeks post-LCMV injection, mice were subjected to permanent ligation of the left anterior descending coronary artery (experimental MI) or a sham surgery and maintained for an additional 3.5 weeks. Mice were maintained on a low-fat semipurified diet from the onset of diabetes. Resident macrophage isolated at the end of the study and adhesion purified for $1 \mathrm{~h}$ before harvest. $A$ : Total and esterified cellular cholesterol $(n=12-20)$. B: Ccl2, II1b, Tnfa, and II6 mRNA ( $n=4-9)$. C: Plasma IL18 levels. D: Quantification of the necrotic cores in the aortic sinus lesion expressed as \% of lesion area. Necrotic cores are defined as acellular areas with cholesterol clefts (indicated by arrows in Fig. 4B) $N=14-24$. $E$ : Quantification of the necrotic cores in the BCA expressed as \% of lesion area. $N=14-22$. F: Viability dye-positive (dead) resident peritoneal macrophages $(n=3-6)$ within the macrophage population $\left(\mathrm{F} 4 / 80\right.$ and $\left.\mathrm{CD} 11 \mathrm{~B}^{+}\right)$, ${ }^{\star} P<0.05$, ${ }^{\star \star \star} P<0.001$, two-way ANOVA $(A, B$, and $F$ ) and three-way ANOVA ( $C-E$; WT vs. KO, nondiabetic vs. diabetic, sham vs. MI significance or as a significant interaction between two conditions [e.g., DxKO indicates that under diabetic conditions there is an effect of the KO, and MlxKO indicates that under MI conditions there is an effect of the KO]) followed by Sidak multiple comparisons tests comparing groups that differ by one parameter (as indicated by lines with asterisks).

to that of an experimental MI. As all our mice underwent surgery, this may explain why we did not observe elevated monocyte levels and, therefore, acceleration of atherosclerosis in our nondiabetic mice subjected to an experimental MI as compared with sham mice. An alternative explanation to the observed differences is the use of APOE-deficient mice compared with our LDLRdeficient model, which is known to augment monocytosis (38). Yet another reason might be differences in infarct size.

It has also been demonstrated that diabetes in mouse models can induce hematopoietic changes that result in monocytosis $(18,25)$. Whether monocytosis or neutrophilia is present and contributes to cardiovascular disease in diabetes in humans is still not fully understood $(18,39,40)$. Similar to in our previous publications $(12,33)$, we did not observe elevated monocyte numbers in this study. The differences in myelopoiesis are most likely due to differences in models and diets used, as cholesterol is a potent driver of monocytosis (41). Here, instead of observing high circulating levels of monocytes, we observed that diabetes suppressed circulating monocyte levels, which was reversed by a deficiency in integrin $\alpha 4$. As integrin $\alpha 4$ is part of the critical monocyte adhesion complex VLA4, it is not surprising that lack of this molecule reduces the emigration of monocytes into tissues and therefore elevates the circulating levels. Accordingly, the deletion of integrin $\alpha 4$ reduces monocyte and neutrophil entry into the peritoneal cavity during sterile inflammation while increasing circulating levels.
Diabetes can result in increased monocyte recruitment in the setting of monocytosis (18). Here we demonstrate that diabetes resulted in increased monocyte recruitment capacity in the absence of monocytosis. This implies that monocytes under diabetes conditions are more likely to migrate into the artery wall, which is consistent with their increased cell surface expression of integrin $\alpha 4$ (Fig. 3B). However, we observed reduced Mac-2 staining within the lesion under diabetes conditions, which might be explained by the drop in circulating monocytes levels. Alternatively, one could speculate that the newly recruited monocytes die shortly after being recruited to the artery wall and therefore do not contribute to lesional Mac-2 staining. This is supported by the findings that diabetes increased macrophage cell death (see Fig. 6F). The increased macrophage cell death, in turn, is consistent with the expanded necrotic cores, as a necrotic core is formed in part due to macrophage death.

Another possibility is that the larger necrotic core in diabetes is a marker of a faster-progressing lesion and not necessarily due to a single mechanism. This is indeed supported by human imaging studies that suggest that diabetes accelerates lesion progression. Won et al. (36) used serial coronary computed tomography angiography to demonstrate that atherosclerotic lesion progression is faster in people with diabetes than in those without. In a similar study, Kim et al. (37) showed that plaque progression, particularly the necrotic core formation and expansion, was accelerated in diabetes. 
In summary, people with diabetes have increased risk of an initial MI but also recurrent MI. Our data would support not the idea that the initial MI directly accelerates atherogenesis but, rather, the idea that diabetes accelerates atherosclerosis. As diabetes is associated with more vessels being affected (42), another lesion becomes the culprit lesion resulting in a subsequent ischemic event.

Acknowledgments. The authors thank Dr. Charles Murry (Department of Pathology, University of Washington) for support and help with the initial study design.

Funding. This study was supported by American Diabetes Association grant 1-16-IBS-153 (to J.E.K.). Part of the study was supported by the Diabetes Research Center at the University of Washington (National Institutes of Health grant P30DK017047).

Duality of Interest. No potential conflicts of interest relevant to this article were reported.

Author Contributions. J.E.K. designed and directed the study. J.E.K. analyzed data and wrote the manuscript. J.E.K. and F.K. performed experiments. A.M.M. assisted with the $\mathrm{Ml}$ and echocardiograms. T.P. provided the $I \operatorname{tga} 4^{f / / f l}$ mice. All authors reviewed the manuscript and provided final approval for submission. J.E.K. is the guarantor of this work and, as such, had full access to all the data in the study and takes responsibility for the integrity of the data and the accuracy of the data analysis.

Prior Presentation. Parts of this study were presented in abstract form at the 79th Scientific Sessions of the American Diabetes Association, San Francisco, CA, 7-11 June 2019.

\section{References}

1. Rawshani A, Rawshani A, Franzén $S$, et al. Risk factors, mortality, and cardiovascular outcomes in patients with type 2 diabetes. N Engl J Med 2018;379: 633-644

2. Sarwar N, Gao P, Seshasai SR, et al.; Emerging Risk Factors Collaboration. Diabetes mellitus, fasting blood glucose concentration, and risk of vascular disease: a collaborative meta-analysis of 102 prospective studies [published correction appears in Lancet 2010;376:958]. Lancet 2010;375:2215-2222

3. Laakso M, Kuusisto J. Insulin resistance and hyperglycaemia in cardiovascular disease development. Nat Rev Endocrinol 2014;10:293-302

4. Juutilainen $A$, Lehto S, Rönnemaa T, Pyörälä K, Laakso M. Type 2 diabetes as a "coronary heart disease equivalent": an 18-year prospective population-based study in Finnish subjects. Diabetes Care 2005;28:2901-2907

5. Liang H, Vallarino C, Joseph G, Manne S, Perez A, Zhang S. Increased risk of subsequent myocardial infarction in patients with type 2 diabetes: a retrospective cohort study using the U.K. General Practice Research Database. Diabetes Care 2014;37:1329-1337

6. Haffner SM, Lehto S, Rönnemaa T, Pyörälä K, Laakso M. Mortality from coronary heart disease in subjects with type 2 diabetes and in nondiabetic subjects with and without prior myocardial infarction. N Engl J Med 1998;339: 229-234

7. Deedwania PC, Ahmed Ml, Feller MA, et al. Impact of diabetes mellitus on outcomes in patients with acute myocardial infarction and systolic heart failure. Eur J Heart Fail 2011;13:551-559

8. Bui QT, Reddy VS, Jacobs JR, et al. Previous myocardial infarction as a risk factor for in-hospital cardiovascular outcomes (from the National Registry of Myocardial Infarction 4 and 5). Am J Cardiol 2013;111:1694-1700

9. Dutta P, Courties G, Wei Y, et al. Myocardial infarction accelerates atherosclerosis. Nature 2012;487:325-329

10. Parathath S, Grauer L, Huang LS, et al. Diabetes adversely affects macrophages during atherosclerotic plaque regression in mice. Diabetes $2011 ; 60$ : 1759-1769
11. Renard CB, Kramer F, Johansson F, et al. Diabetes and diabetes-associated lipid abnormalities have distinct effects on initiation and progression of atherosclerotic lesions. J Clin Invest 2004;114:659-668

12. Kanter JE, Shao B, Kramer F, et al. Increased apolipoprotein C3 drives cardiovascular risk in type 1 diabetes. J Clin Invest 2019;129:4165-4179

13. Johansson F, Kramer F, Barnhart S, et al. Type 1 diabetes promotes disruption of advanced atherosclerotic lesions in LDL receptor-deficient mice. Proc Natl Acad Sci U S A 2008;105:2082-2087

14. Virmani R, Burke AP, Kolodgie F. Morphological characteristics of coronary atherosclerosis in diabetes mellitus. Can J Cardiol 2006;22(Suppl. B):81B-84B 15. Kanter JE, Kramer F, Barnhart $\mathrm{S}$, et al. Diabetes promotes an inflammatory macrophage phenotype and atherosclerosis through acyl-CoA synthetase 1. Proc Natl Acad Sci U S A 2012;109:E715-E724

16. Moreno PR, Murcia AM, Palacios IF, et al. Coronary composition and macrophage infiltration in atherectomy specimens from patients with diabetes mellitus. Circulation 2000;102:2180-2184

17. Lamharzi N, Renard CB, Kramer F, et al. Hyperlipidemia in concert with hyperglycemia stimulates the proliferation of macrophages in atherosclerotic lesions: potential role of glucose-oxidized LDL. Diabetes 2004;53:3217-3225

18. Nagareddy PR, Murphy AJ, Stirzaker RA, et al. Hyperglycemia promotes myelopoiesis and impairs the resolution of atherosclerosis. Cell Metab 2013;17: 695-708

19. Richardson M, Hadcock SJ, DeReske M, Cybulsky MI. Increased expression in vivo of VCAM-1 and E-selectin by the aortic endothelium of normolipemic and hyperlipemic diabetic rabbits. Arterioscler Thromb 1994;14:760-769

20. Ribau JC, Hadcock SJ, Teoh K, DeReske M, Richardson M. Endothelial adhesion molecule expression is enhanced in the aorta and internal mammary artery of diabetic patients. J Surg Res 1999;85:225-233

21. Rask-Madsen C, Li Q, Freund B, et al. Loss of insulin signaling in vascular endothelial cells accelerates atherosclerosis in apolipoprotein $\mathrm{E}$ null mice. Cell Metab 2010;11:379-389

22. Cipolletta C, Ryan KE, Hanna EV, Trimble ER. Activation of peripheral blood CD14+ monocytes occurs in diabetes. Diabetes 2005;54:2779-2786

23. Patel SS, Thiagarajan R, Willerson JT, Yeh ET. Inhibition of alpha4 integrin and ICAM-1 markedly attenuate macrophage homing to atherosclerotic plaques in ApoE-deficient mice. Circulation 1998;97:75-81

24. Huo Y, Hafezi-Moghadam A, Ley K. Role of vascular cell adhesion molecule-1 and fibronectin connecting segment-1 in monocyte rolling and adhesion on early atherosclerotic lesions. Circ Res 2000;87:153-159

25. Ulyanova T, Priestley GV, Banerjee ER, Papayannopoulou T. Unique and redundant roles of alpha4 and beta2 integrins in kinetics of recruitment of lymphoid vs myeloid cell subsets to the inflamed peritoneum revealed by studies of genetically deficient mice. Exp Hematol 2007;35:1256-1265

26. Scott LM, Priestley GV, Papayannopoulou T. Deletion of alpha4 integrins from adult hematopoietic cells reveals roles in homeostasis, regeneration, and homing. Mol Cell Biol 2003;23:9349-9360

27. Neidig LE, Weinberger F, Palpant NJ, et al. Evidence for minimal cardiogenic potential of stem cell antigen 1-positive cells in the adult mouse heart. Circulation 2018;138:2960-2962

28. Virag Jl, Murry CE. Myofibroblast and endothelial cell proliferation during murine myocardial infarct repair. Am J Pathol 2003;163:2433-2440

29. Li X, Gonzalez 0 , Shen X, et al. Endothelial acyl-CoA synthetase 1 is not required for inflammatory and apoptotic effects of a saturated fatty acid-rich environment. Arterioscler Thromb Vasc Biol 2013;33:232-240

30. Gerhardt T, Ley K. Monocyte trafficking across the vessel wall. Cardiovasc Res 2015;107:321-330

31. Boudina S, Abel ED. Diabetic cardiomyopathy revisited. Circulation 2007; 115:3213-3223

32. Abram CL, Roberge GL, Hu Y, Lowell CA. Comparative analysis of the efficiency and specificity of myeloid-Cre deleting strains using ROSA-EYFP reporter mice. J Immunol Methods 2014;408:89-100 
33. Vallerie SN, Kramer F, Barnhart S, et al. Myeloid cell prostaglandin E2 receptor EP4 modulates cytokine production but not atherogenesis in a mouse model of type 1 diabetes. PLoS One 2016;11:e0158316

34. Hoffmann J, Ospelt M, Troidl C, et al. Sham surgery and inter-individual heterogeneity are major determinants of monocyte subset kinetics in a mouse model of myocardial infarction. PLoS One 2014;9:e98456

35. Tacke F, Alvarez D, Kaplan TJ, et al. Monocyte subsets differentially employ CCR2, CCR5, and CX3CR1 to accumulate within atherosclerotic plaques. J Clin Invest 2007;117:185-194

36. Won KB, Lee SE, Lee BK, et al. Longitudinal assessment of coronary plaque volume change related to glycemic status using serial coronary computed tomography angiography: a PARADIGM (Progression of AtheRosclerotic PIAque Determlned by Computed TomoGraphic Angiography Imaging) substudy. J Cardiovasc Comput Tomogr 2019;13:142-147

37. Kim U, Leipsic JA, Sellers SL, et al. Natural history of diabetic coronary atherosclerosis by quantitative measurement of serial coronary computed tomographic angiography: results of the PARADIGM study. JACC Cardiovasc Imaging 2018;11:1461-1471
38. Murphy AJ, Akhtari M, Tolani S, et al. ApoE regulates hematopoietic stem cell proliferation, monocytosis, and monocyte accumulation in atherosclerotic lesions in mice. J Clin Invest 2011;121:4138-4149

39. Valle A, Giamporcaro GM, Scavini M, et al. Reduction of circulating neutrophils precedes and accompanies type 1 diabetes. Diabetes 2013;62:20722077

40. Kanter JE, Hsu CC, Bornfeldt KE. Monocytes and macrophages as protagonists in vascular complications of diabetes. Front Cardiovasc Med 2020;7:10

41. Murphy AJ, Dragoljevic D, Tall AR. Cholesterol efflux pathways regulate myelopoiesis: a potential link to altered macrophage function in atherosclerosis. Front Immunol 2014;5:490

42. Rana JS, Dunning A, Achenbach S, et al. Differences in prevalence, extent, severity, and prognosis of coronary artery disease among patients with and without diabetes undergoing coronary computed tomography angiography: results from 10,110 individuals from the CONFIRM (COronary CT Angiography EvaluatioN For Clinical Outcomes): an InteRnational Multicenter Registry. Diabetes Care 2012; 35:1787-1794 